Joanna L. Kramer*, DO, MPH and Kathleen De Asis, DO, MS

\title{
Osteopathic interventions via telehealth in a pediatric population: a retrospective case series
}

https://doi.org/10.1515/jom-2021-0124

Received April 21, 2021; accepted June 22, 2021;

published online August 19, 2021

\section{Abstract}

Context: Healthcare delivery was dramatically affected during the coronavirus disease 2019 (COVID-19) pandemic. Many outpatient visits were cancelled or forgone for fear of exposure to the virus, allowing telemedicine to take on a much larger role in healthcare. The delivery of manual therapies, such as osteopathic manipulative treatment (OMT), via telehealth posed a unique challenge as these are typically provided in-person by a trained osteopathic physician. This study provides a description of one osteopathic pediatrician's experience in delivering osteopathic interventions to pediatric patients via telehealth. To our knowledge, these techniques have not previously been described in the literature.

Objectives: To detail the experience of one osteopathic pediatrician's experience in delivering osteopathic interventions via telehealth.

Methods: Patients were offered the option of converting their existing OMT appointment to a telehealth visit. Prior to the appointment, instructions were emailed to the patient's parent or guardian along with a voluntary survey to provide feedback. Thirty-minute telehealth visits were conducted during which the provider gave verbal and visual instructions to a parent or guardian over a video platform to guide them in providing treatment to the patient based on osteopathic principles. Patients aged 3 and older rated their pain before and after the appointment using the Wong-Baker FACES scale. Deidentified patient demographics, chief complaints, treatments, anatomic locations, and pain scores were recorded in a REDcap database. Descriptive statistics were analyzed and paired

\footnotetext{
*Corresponding author: Joanna L. Kramer, DO, MPH, Phoenix Children's Hospital Division of Primary, Complex Care, and Adolescent Medicine, Ambulatory Building 2nd Floor, 1919 E. Thomas Road, Phoenix, AZ 85016, USA, E-mail: joanna.kramer22@gmail.com Kathleen De Asis, DO, MS, Phoenix Children's Pediatric Residency Program Alliance, Phoenix, AZ, USA
}

samples t-tests were used with a p-value of $<0.05$ used to determine significance.

Results: Eighteen patients ranging from 6 months to 19 years of age were treated utilizing osteopathic interventions via telehealth during 54 distinct visits. The most common chief complaints treated were back $(n=31$; $26.3 \%)$ and neck $(n=28 ; 23.7 \%)$ pain. The most common osteopathic techniques upon which instruction was based were inhibition $(n=131 ; 29.7 \%)$ soft tissue $(n=127 ; 28.8 \%)$ and counterstrain $(\mathrm{n}=78 ; 17.7 \%)$. The average posttreatment pain score (2.57) was significantly lower than the average pre-treatment pain score (6.77) $\mathrm{p}<0.01$. No serious complications were observed.

Conclusions: In our small retrospective case series, osteopathic interventions via telehealth resulted in decreased average pain scores following treatment while minimizing risk of viral exposure and transmission. Further study is needed to determine if such treatment methods could be effective on a larger scale when distance or illness preclude an in-person OMT visit.

Keywords: OMT; osteopathic; pediatrics; telehealth; telemedicine.

Osteopathic manipulative treatment (OMT) is a set of hands-on techniques often utilized in the pediatric population to optimize anatomic structure and improve physiologic function. OMT is performed by osteopathic physicians and requires years of training to develop and refine. However, these manual techniques often require prolonged physical contact between patient and practitioner. During the coronavirus disease 2019 (COVID-19) pandemic there was concern that such contact could increase the risk of viral exposure and transmission either to or from a patient $[1,2]$, in addition to the risk of exposure to other patients in a medical office. Multiple studies have shown an increase in delayed or canceled routine medical care during the COVID-19 pandemic, which could lead to poorer health outcomes [3, 4]. For example, one paper published in Morbidity and Mortality Weekly Reports (MMWR) surveyed 4,975 US adults and reported that $40.9 \%$ of US adults have avoided or delayed medical care due to concerns of COVID-19 [5]. Another paper in MMWR surveyed 9,539 
children in Michigan and revealed a substantial decline in upto-date vaccination status among children aged 5 months, from 66 to $67 \%$ pre-pandemic to $49.7 \%$ in May 2020 [6].

Telemedicine is one way that healthcare providers were able to continue to provide essential care to patients during the pandemic [7]. Early in the pandemic, our academic pediatric practice in Phoenix, AZ, attempted to provide as much care as possible via telemedicine to avoid potential virus exposure in waiting rooms and examination rooms. Therefore, we implemented a trial of osteopathic interventions performed by a parent or guardian on the pediatric patient via telemedicine with one osteopathic physician's instruction, supervision, and guidance over video. Weighing risks and benefits during the pandemic, we hypothesized that guiding parents through simplified techniques under careful guidance could provide modest or even significant benefit to patients without increasing the risk of viral transmission as would an in-person visit.

This retrospective case series examines the demographics, conditions, and techniques utilized for patients who were treated with osteopathic interventions via telehealth during the COVID-19 pandemic in an academic osteopathic pediatric practice in Phoenix, AZ. Pre- and post-treatment pain scales are compared to demonstrate efficacy, complications are examined, and patient perceptions from a voluntary survey are considered. The aim of the study is to demonstrate our experience with osteopathic interventions via telehealth and perhaps create an avenue for patient treatment when distance, illness, or other factors prohibit an in-person OMT visit. No such study presently exists in the literature to our knowledge.

\section{Methods}

Institutional review board (IRB) exemption and waiver of informed consent was obtained through the Phoenix Children's Hospital's IRB (\#20-126). No funding was received, and patients did not receive compensation for this retrospective study.

Existing patients of one osteopathic pediatrician (J.K.) in Phoenix, $\mathrm{AZ}$, were offered the option of converting their previously scheduled inperson OMT appointment to a visit utilizing telehealth during the COVID-19 pandemic (from March-October 2020). If the parent/guardian elected to convert the appointment to telehealth, instructions for optimal device setup and treatment positioning were emailed ahead of time as well as an anonymous voluntary survey to provide feedback on the experience. During 30-min appointments, adult parents or family members were guided by the osteopathic physician through basic techniques grounded in osteopathic principles to treat patients over a video platform. The osteopathic physician verbally and visually instructed parents on patient positioning, hand positioning, palpatory changes, and manual manipulation based in osteopathic treatment and principles. Patients aged 3 and older were asked to rate their pain at the beginning and end of each session using the Wong-Baker FACES scale.
For example, to demonstrate suboccipital inhibition the osteopathic physician would instruct the parent "Have your child lay on his/her back with his/her head toward you. Place your fingers underneath the patient's head and slide them down the back of the head to the spot where the bony skull ends and the muscle of the neck begins. Hinge your fingers at the knuckle joint to create a bridge for the patient's neck on your fingertips, with fingertips pointing up to the ceiling. Ask your child to relax his/her head and support the weight of the patient's neck on your fingertips. You should feel the head getting heavier into the palms of your hands as the trapezius muscle relaxes." The osteopathic physician would concurrently demonstrate the hand position or treatment over the video screen. When a resident or student learner was present and willing to participate, he/she would demonstrate ideal patient position while the physician demonstrated the treatment using the learner as a model.

Deidentified data were compiled into a REDcap database consisting of demographics, chief complaint, treatments, anatomic locations, and pre- and post-treatment pain scales. Paired samples t-tests were used to determine efficacy with a statistical significance denoted by a p-value of $<0.05$.

\section{Results}

A total of 18 patients were treated with osteopathic interventions via telehealth during 54 separate visits. The number of appointments ranged from 1 to 9 visits per patient (mean, 3 sessions; median, 2 sessions) (Table 1). Twelve females and six males were treated (Table 1). The patients' ages ranged from 6 months to 19 years, with an average age of 13.6 years (Table 1). Data on race and ethnicity were not available as this was a retrospective study. Back $(n=31 ; 26.3 \%)$ and neck $(\mathrm{n}=28 ; 23.7 \%)$ pain were the most common chief complaints treated followed by head ( $\mathrm{n}=17 ; 14.4 \%)$, upper extremity $(\mathrm{n}=16 ; 13.6 \%)$, lower extremity $(\mathrm{n}=15 ; 12.7 \%)$, abdominal $(\mathrm{n}=7 ; 5.9 \%)$, hip $(\mathrm{n}=3 ; 2.5 \%)$, and rib $(\mathrm{n}=1 ; 0.8 \%)$ pain (Figure 1). Video guidance was provided to a parent or adult relative by one osteopathic pediatrician based on osteopathic techniques. Inhibition ( $\mathrm{n}=131 ; 29.7 \%)$, soft tissue $(\mathrm{n}=127$; $28.8 \%)$, and counterstrain $(n=78 ; 17.7 \%)$ techniques were the most common upon which interventions were based

Table 1: Demographics of patients receiving osteopathic interventions via telehealth.

\begin{tabular}{lr}
\hline Gender, no. of patients & \\
Female & 12 \\
Male & 6 \\
Age, years & \\
Mean & 13.6 \\
Median & 15 \\
Range & $0.5-19$ \\
No. of visits & \\
Mean & 3 \\
Median & 2 \\
Range & $1-9$ \\
\hline
\end{tabular}




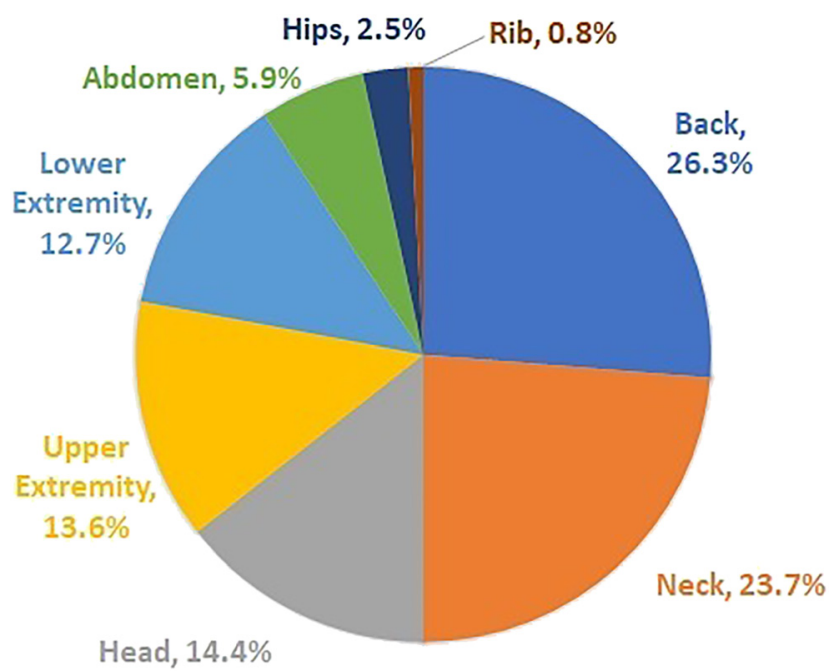

Figure 1: Chief pain complaints treated with osteopathic interventions via telehealth.

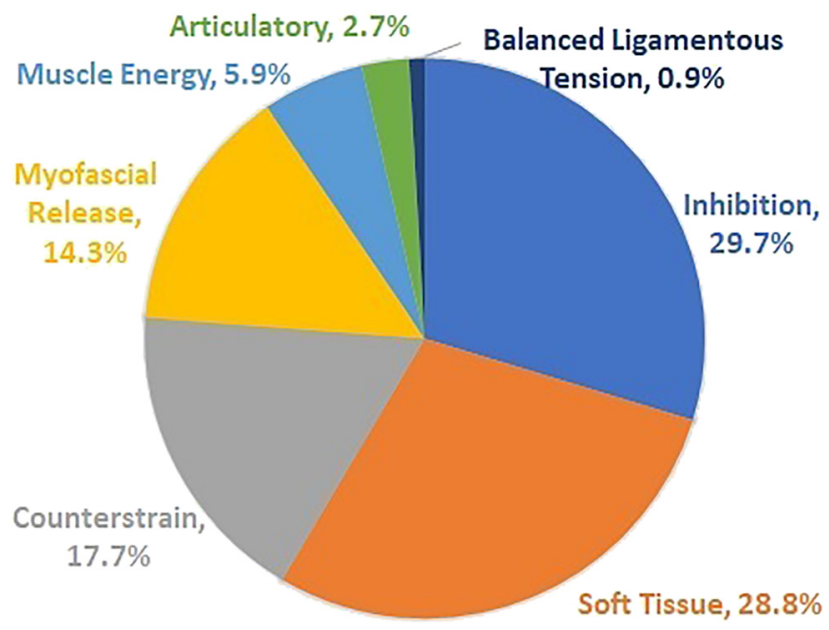

Figure 2: Techniques upon which instruction was based during osteopathic interventions via telehealth.

(Figure 2). We also provided instruction based upon osteopathic principles utilized in the techniques of myofascial release $(n=63 ; 14.3 \%)$, muscle energy $(n=26 ; 5.9 \%)$, articulatory $(\mathrm{n}=12 ; 2.7 \%)$, and balanced ligamentous tension $(\mathrm{n}=4$; $0.9 \%$ ) techniques (Figure 2). Technique percentages were calculated based on the number of times the treatment was utilized during any visit (counted more than once if performed on multiple body sites) out of the total number of techniques performed in all visits.

We compared the reduction in pain scores during telehealth visits to the most recent in-person visit for the same subset of patients and found that there was no significant difference in pain reduction. The average pre-treatment pain score was $6.77 \pm 2.07$ and the average post-treatment score was $2.57 \pm 2.58$ ( $<<0.01)$ for telehealth appointments

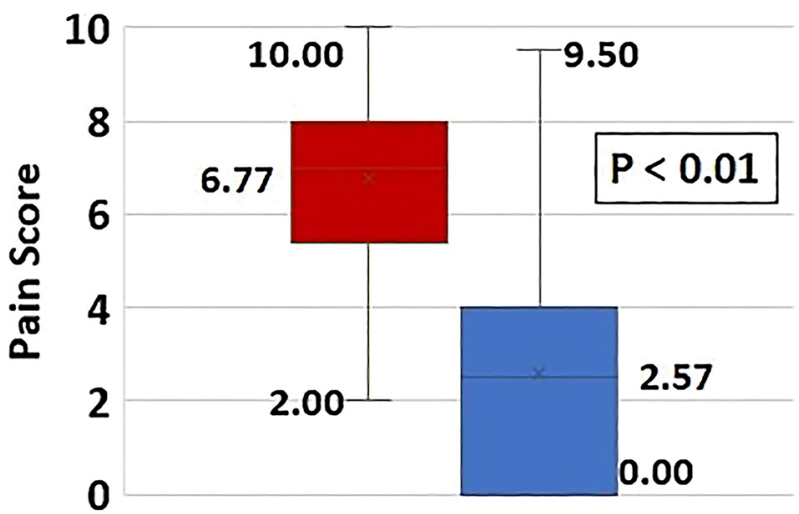

- Pre-Treatment $\square$ Post-Treatment

Figure 3: Comparison of pre- and post-treatment pain scores during osteopathic interventions via telehealth.

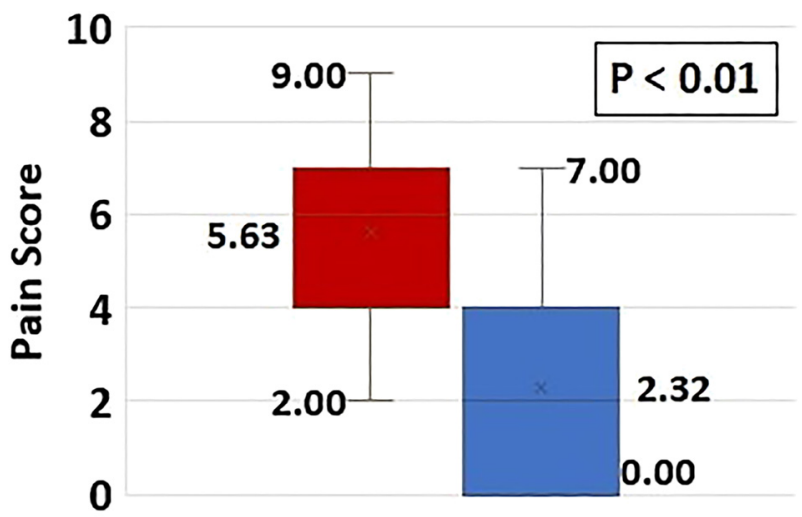

- Pre-Treatment $\|$ Post-Treatment

Figure 4: Comparison of pre- and post-treatment pain scores during osteopathic interventions performed in-person.

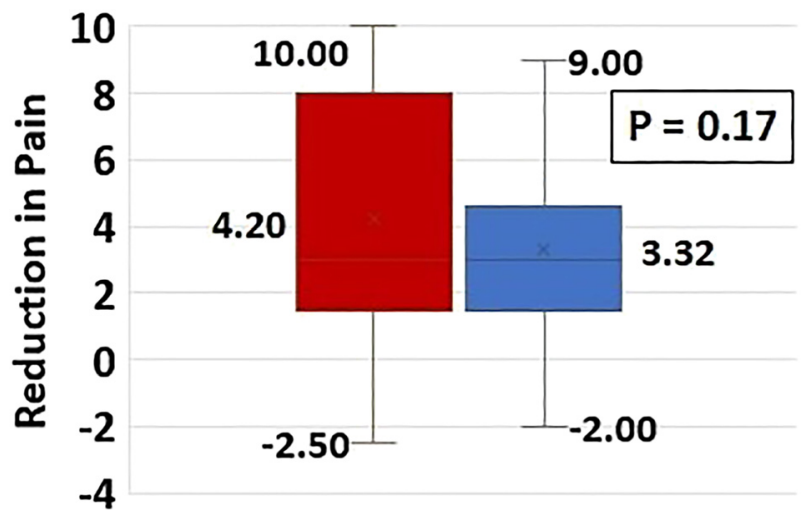

\section{- Telehealth $₫ \ln$ Person}

Figure 5: Reduction in pain scores during telehealth vs. in-person visits. 
(Figure 3). The average pain score pre- and post-treatment for in-person osteopathic interventions were $5.63 \pm 1.96$ and $2.32 \pm 2.26$, respectively $(\mathrm{p}<0.01)$ (Figure 4). The average improvement of Wong-Baker FACES scores for telehealth treatments were $4.20 \pm 3.42$ and for in-person visits were $3.32 \pm 2.61(\mathrm{p}=0.17)$ (Figure 5). There were no serious complications reported from the osteopathic interventions. Four patients during 5 of the 54 total visits (9.3\%) had mild temporary worsening of pain following osteopathic interventions via telehealth. Worsening of pain resolved the same day of the treatment for all 5 visits.

Five parents or guardians responded to the survey via a deidentified survey software platform (Appendix). Of these, 4 responded that they were either somewhat or very uncomfortable performing osteopathic interventions prior to the telehealth visit. After the telehealth visit, all 5 responded that they were somewhat or very comfortable performing osteopathic interventions. Three parents or guardians responded that they would prefer in-person visits over telehealth visits in the future, if available. One responded that they would prefer telehealth visits and one did not respond to the question.

\section{Discussion}

At the onset of the pandemic, we did not imagine that osteopathic interventions via telehealth would have been possible, much less effective, at relieving symptoms. However, telehealth infrastructure was rapidly developed at our academic institution as a complete shutdown was not an option. Once this framework was available, some families were amenable and even excited to attempt these techniques at home. Prior to the pandemic, we often instructed parents in some basic osteopathic techniques (e.g., Galbreath maneuver or suboccipital inhibition) to assist in treating their child at home and these families were willing to learn some additional techniques. However, instructing parents over a telehealth platform was certainly more challenging than instructing them in-person. In person the doctor can more easily guide the parent's hand position and monitor force. Additionally, the patient can provide feedback to the parent as to whether the treatment feels similar when the parent performs it vs. when the doctor performs it. However, with no other options at the time, we trialed the telehealth interventions with a select few patients and parents who were very familiar with OMT. We were so impressed by their success and desire to schedule additional telehealth OMT appointments that we decided to share our experience in a retrospective case series. Prior to telehealth we commonly instructed parents in inhibition techniques so it is not surprising that these were more easily adapted to the telehealth platform. Soft tissue and counterstrain techniques were also relatively easy to instruct via telehealth. Some of the osteopathic techniques upon which treatment was based (e.g., myofascial release and balanced ligamentous tension) require advanced palpatory skills that would not have been developed in a non-physician parent or family member performing the interventions. We are not suggesting the parent was performing these techniques precisely as a trained osteopathic physician could, but that the provider was instructing on simplified methods using the theory behind them and that vocabulary does not exist to describe these otherwise. Of note, we billed only a CPT code and did not apply a procedure code for these visits as the treatments were not physically performed by a licensed provider.

For families, patients, and this provider, osteopathic interventions via telehealth were a way to deliver care to a fragile pediatric population without increasing the risk of viral exposure and transmission to patients or medical staff during the early and uncertain stages of the COVID-19 pandemic. Furthermore, many healthcare providers and practices suffered monetary losses due to forgone medical care during the COVID-19 pandemic. A study conducted by the Medical Group Management Association revealed that $97 \%$ of 724 medical practices surveyed have experienced negative financial impacts due to COVID-19 [8]. This provider found that osteopathic interventions via telehealth were not only beneficial to the patients but also a way to continue a revenue stream to support the practice.

For our small sample size, we were able to show a statistically significant decrease in average pain scores after the application of osteopathic interventions via telehealth. Additionally, comments from patient surveys included themes that families appreciated the safety and flexibility of completing these appointments from home to avoid viral exposure as well as learning strategies to help alleviate their child's symptoms both during and after the appointment. No serious complications were reported from the osteopathic interventions via telehealth. Four patients during 5 visits (9.3\%) noted mild temporary worsening of pain following osteopathic interventions. This is slightly higher than the rate reported in the literature for in-person OMT visits (2.5\%) [9] and might be attributed to parental misapplication of technique or force. Performing OMT in a pediatric population can be challenging even in an inperson environment because certain techniques require the patient to be able to reliably follow instructions and provide input. Considering the added challenge of a video platform, we experientially felt that older patients tended 
to tolerate and respond better to osteopathic interventions via telehealth.

Other challenges we encountered delivering osteopathic interventions over telehealth included the use of interpreters for non-English speakers. The use of an interpreter over telehealth was an additional layer of complexity which proved difficult to navigate when instructing techniques through a 3rd party. If a student or resident learner was present for the visits (as is often the case in this academic institution), it was helpful for the physician to demonstrate the technique on the learner over video so families could visualize proper technique for both English and non-English speakers.

This study was limited by its retrospective nature and small sample size. Given the relatively quick change within our practice from all in-person visits to the majority telehealth visits, a randomized controlled trial was not feasible at the time. The pandemic created a unique situation where large numbers of patients either preferred or were required to receive care over telehealth. An additional limitation of this study is that replicating it on a larger scale may be difficult as most patients are now opting to schedule in-person OMT visits. Nonetheless, we feel that this experience is valuable to share for circumstances when an in-person OMT visit is not possible as well as to illustrate the impact of the COVID-19 pandemic on the practice of OMT.

\section{Conclusions}

The COVID-19 pandemic has drastically altered healthcare delivery. Many patients went without routine healthcare and manual therapies during this time for a variety of reasons. It is difficult if not impossible to measure the impact of this foregone healthcare. Though OMT is a complex set of skills that requires years of training, our experience aims to show that osteopathic principles can be applied to help deliver care in a safe and effective manner over telehealth when the risks outweigh the benefits of an in-person appointment. This could create avenues for treatments based in osteopathic philosophy when distance, illness, or other factors prohibit an in-person appointment.

Acknowledgments: The authors would like to thank Ted Swing, PhD, for providing input on study design and data analysis and our patients and their families for providing inspiration for the study.

Research funding: None reported.
Author contributions: Both authors provided substantial contributions to conception and design, acquisition of data, or analysis and interpretation of data; both authors drafted the article or revised it critically for important intellectual content; both authors gave final approval of the version of the article to be published; and both authors agree to be accountable for all aspects of the work in ensuring that questions related to the accuracy or integrity of any part of the work are appropriately investigated and resolved.

Competing interests: None reported.

Ethical approval: This study was deemed exempt from Institutional Review Board (IRB) review and a waiver of informed consent was granted through Phoenix Children's Hospital IRB (\#20-126).

\section{References}

1. Shaukat N, Ali DM, Razzak J. Physical and mental health impacts of COVID-19 on healthcare workers: a scoping review. Int J Emerg Med 2020;13:40.

2. Lau J, Tan DHY, Wong GJ, Lew YJ, Chua YX, Low LL, et al. Prepared and highly committed despite the risk of COVID-19 infection: a cross-sectional survey of primary care physicians' concerns and coping strategies in Singapore. BMC Fam Pract 2021;22:22.

3. Negrini S, Grabljevec K, Boldrini P, Kiekens C, Moslavac S, Zampolini $\mathrm{M}$, et al. Up to 2.2 million people experiencing disability suffer collateral damage each day of COVID-19 lockdown in Europe. Eur J Phys Rehabil Med 2020;56:361-5.

4. Goulabchand R, Claret PG, Lattuca B. What if the worst consequences of COVID-19 concerned non-COVID patients? J Infect Public Health 2020;13:1237-9.

5. Czeisler ME, Marynak K, Clarke KEN, Salah Z, Shakya I, Thierry JM, et al. Delay or avoidance of medical care because of COVID19-related concerns - United States, June 2020. MMWR Morb Mortal Wkly Rep 2020;69:1250-7.

6. Bramer CA, Kimmins LM, Swanson R, Kuo J, Vranesich P, JacquesCarroll LA, et al. Decline in child vaccination coverage during the COVID-19 pandemic - Michigan Care Improvement Registry, May 2016-May 2020. MMWR Morb Mortal Wkly Rep 2020;69:630-1.

7. Bokolo A. Use of telemedicine and virtual care for remote treatment in response to COVID-19 pandemic. J Med Syst 2020;44:132.

8. Medical Group Management Association. COVID-19 Financial Impact on Medical Practices. https://www.mgma.com/ getattachment/9b8be0c2-0744-41bf-864f-04007d6adbd2/ 2004-G09621D-COVID-Financial-Impact-One-Pager-8-5x11-MW-2. pdf.aspx?lang=en-US\&ext=.pdf [Accessed 31 Mar 2021].

9. Degenhardt BF, Johnson JC, Brooks WJ, Norman L. Characterizing adverse events reported immediately after osteopathic manipulative treatment. J Am Osteopath Assoc 2018;118:141-9.

Supplementary Material: The online version of this article offers supplementary material (https://doi.org/10.1515/jom-2021-0124). 